\title{
The Characteristics of Peripheral Blood Mononuclear Cells in Takayasu Arteritis by Single Cell RNA Sequencing
}

\author{
Qing $\mathrm{Gao}^{1}$, Jinge $\mathrm{Yu}^{2}$, Zuoguan Chen ${ }^{1}$, Yongpeng Diao ${ }^{1}$, Yuqing Miao ${ }^{1}$, Jinfeng Yin ${ }^{3}$, \\ Junnan $\mathrm{Jia}^{3}$, Weimin $\mathrm{Li}^{3}$, and Yongjun $\mathrm{Li}^{1}$ \\ ${ }^{1}$ Beijing Hospital \\ ${ }^{2}$ Renmin University of China \\ ${ }^{3}$ Capital Medical University
}

January 28, 2021

\begin{abstract}
Objectives Takayasu Arteritis (TA) is a rare non-specific vascular inflammation and has deleterious effects on patients' health. Recent studies have advanced in TA diagnosis and treatment, but the research on the immune cell atlas of peripheral blood is still less. For this purpose, we performed single-cell RNA sequencing (scRNA-seq) to analyze the inflammatory cell types and cell markers in TA patients' Peripheral blood mononuclear cells (PBMCs). Methods 4 TA patients and 4 health controls were enrolled in our study from 2019.10 to 2020.5. Their PBMCs samples were collected and performed scRNA-seq. We used Seurat package (v.3.2.2) in R studio (v.3.5.3) for data analysis, and ?2 tests were applied for comparing the composition ratio of each cell type by SPSS 20.0. Results CD14+ monocytes, GZMB + NKT cells, CD56dim CD16+ NK cells, and naive B cells were significantly increased in TA patients as compared to healthy controls and the expression of THBS1, CD163, AREG, IFITM1, TXNIP, and IGHGs was elevated in the peripheral blood of TA patients. Conclusion Except CD4+ T cells, monocytes, NK cells, NKT cells, B cells also play an important role in TA pathogenesis. The elevated markers have different functions in different types of PBMCs, and they can be used as potential diagnostic markers for TA diagnosis.
\end{abstract}

\section{Introduction}

Takayasu Arteritis (TA) is a rare, non-specific vascular inflammation involving large arteries and their main branches. The disorder occurs worldwide, but is most prevalent in East Asia. In addition, the disease progresses rapidly after the onset and relapses/recurring cycles of inflammation affect patient physiology and psychology significantly. Few symptoms show in the early stage make this disease hardly to be discovered in time. Physical examination along with imaging and serological tests is used to assess the extent of inflammation and progression in $\mathrm{TA}(1)$.

The most commonly used drugs for treating TA are glucocorticoids(2), methotrexate(3), and mycophenolate mofetil $(4,5)$, however, relapses are frequent. In recent years, biological agents such as Tocilizumab (TCZ)(6) and Abatacept (ABA)(7) are being explored as alternative treatments for TA. Patients with poor response to standard medications and vascular blockage require surgical intervention involving endovascular management and open surgical repair $(\mathrm{OSR})(8,9)$. However, surgical intervention does not prevent long-term restenosis and new lesions due to disease recurrence(10-12). To further improve the treatment strategy and prognosis of TA patients, research on its pathogenesis has become the focus of TA-related research.

Recent studies have focus on the significant role of $\mathrm{CD} 4^{+} \mathrm{T}$ cell and IL-6 signaling pathway in development and progression of TA(13-15). Although several groups have studied gene mutations and protein expression in TA patients. However, the relationship between these two is rarely mentioned, which is due to the less data on the types and transcriptomes of peripheral blood cells. 
Single-cell RNA sequencing (scRNA-seq) is a high-throughput technology based on intracellular transcriptome sequencing to explain the diseases at the cellular level $(16,17)$, which give us a chance to find new cell types and cell functions that have not been discovered before. This new technology is being widely used in human immune and cardiovascular diseases $(18,19)$. In this study, single cell RNA sequencing (scRNA-seq) was used to dissect the transcriptomes of peripheral blood mononuclear cells (PBMCs) in TA patients in order to better understand to this disease.

\section{Material and method}

\section{Patients and clinical data}

This study included four TA patients admitted to our hospital from 2019.10 to 2020.5. We collected peripheral blood samples from the patients. All samples were used for scRNA-seq. In addition, peripheral blood from four age-sex matched healthy individuals was collected as control for scRNA-seq. All the contents of this study meet the relevant requirements of the ethics committee. Written consent was obtained from the patients/healthy individuals or their families

The basic clinical data obtained from TA patients are described inTable S1. The four female patients were aged between 16 - 39 years, which is in line with the age and gender prevalence of TA. Although the C-reactive protein (CRP) and erythrocyte sedimentation rate (ESR) of the patients were within the normal range, the history of taking glucocorticoids (methylprednisolone or prednisone acetate) and other drugs (methotrexate) prevented these patients from being rated on NIH scores.

\section{Single-cell suspensions of PBMC preparation}

Density gradient centrifugation method was utilized to obtain PBMCs. We used PBS (Solarbio, P1022-500) to dilute the whole blood sample at a ratio of $1: 1$, and then slowly added the diluted whole blood sample to approximately 2/3 of the volume of Ficoll (GE Healthcare, 17-1440-02). After centrifugation at $400 \mathrm{~g}$ for 35 min, three layers were obtained based on size and density. The middle cell suspension layer was taken into a new 15-mL centrifuge tube and was filled with PBS and centrifuged again at $300 \mathrm{~g}$ for $7 \mathrm{~min}$, supernatant was discarded and the pellet containing PBMCs were washed twice, and the final sample was resuspended in PBS to obtain a final concentration of $1 \times 10^{5}$ cells $/ \mathrm{mL}$. Viability staining with $0.4 \%$ Trypan blue solution (Sigma, T8154) was performed and viable cells were counted under the microscope.

\section{Single cell RNA sequencing}

Single-cell suspensions were then loaded onto microfluidic devices and scRNA-seq libraries were constructed according to Singleron GEXSCOPETM protocol by GEXSCOPETM Single-Cell RNA Library Kit (Singleron Biotechnologies). Individual libraries were diluted to $4 \mathrm{nM}$ and pooled for sequencing. Pools were sequenced on an Illumina HiSeq X with 150 bp paired-end reads.

\section{Single-cell data quality control, processing and analysis}

The Seurat package (v.3.2.2)(70, 71) was applied for quality control, processing, and analysis. Each Seurat object was created with genes that were expressed in more than three cells. Quality control conditions were set as: (1) only genes within 200 and 3000; (2) the percentage of mitochondrial genes less than $20 \%$ were included for downstream analysis(Figure S5a). After QC, the remaining cells were used for further analysis, as shown in Table S2 . A total of 29942 cells were included in the computational analysis. Among them, the case group (TA) had 8966 cells and the healthy control group (HC) had 20976 cells.

The CellcycleScoring function was applied to mitigate the possible influence of cell cycles. SCTransform was adopted by default to reduce potential batch effects or technical variations. PCA was set at 1:20, and then unsupervised cell clustering was performed (Figure S5b) .

Find All Markers by default methods was used to obtain the DEGs specifically in each cluster, and the representative markers (genes with the highest avg_logFC and adjusted $\mathrm{p}$ value $<0.05$ ) were then chosen 
for cluster labeling. DEGs between TA samples and healthy controls in each cluster were identified with function Find Markers.

In order to count the differences in cell composition between the TA group and the HC group, we used the $\chi^{2}$ analysis method in SPSS 20.0 to analyze the differences in the composition ratios of various cell types, and cells with higher composition ratios in the TA group were used.

\section{Statistical analysis}

We used $\chi^{2}$ tests to analyze the composition ratio of M, T, and B cell populations by SPSS 20.0. Results were considered significant when $\mathrm{P}<0.05$.

\section{Result}

\section{Identification of major PBMC subtypes by scRNA-seq}

We analyzed the PBMCs from four TA patients and four healthy controls (HC). After quality control, 8966 cells from the TA patients and 20976 cells from the HC group were further analyzed by scRNA-seq. After analysis, five major cell clusters were identified, as shown inFigure 1a and Table 1, including M cells (Cluster 0,4,6,8), T/NK cells (Cluster 1,2), B cells (Cluster 3,9), megakaryocytes (Cluster 7) and unknown subset (Cluster 5).

The high expression of S100A8, LYZ, and S100A9 (Figure 1b, d)suggested that clusters 0, 4, 6, 7, and 8 are antigen-presenting cells (APCs). High expression of PF4 and PPBP (Figure 1d), suggested cluster 7 to be megakaryocytes. Low CD14 (Figure 1c) and high HLA gene expression (Figure 1d) suggested cluster 8 to be dendritic cells (DCs). Both cluster 1 and cluster 2 were identified as T/NK cells, because of the high expression levels of CD2, CD3D, NKG7, and GNLY (Figure 1b, c, d) . MS4A1, CD79A, and CD79B in cluster 3 and ITM2C, TCF4, and CCDC50 in cluster 9 (Figure 1b, $\mathbf{c}, \mathbf{d}$ )indicate that both of them are B cells. Cluster 5, with high expression level of NEAT1 and XIST, is still unknown.

We broadly classified the clusters under three major cell types mainly, M (Cluster 0, 4, 6, 8), T cells (Cluster 1, 2), and B cells (Cluster 3,9).

\section{Monocyte/Macrophage/DC is the largest cell population in PBMCs}

Monocyte/Macrophage/DC (Cluster 0, 4, 6, and 8) accounted for 50.7\% (15186 cells), highest proportion of all cell types. After unsupervised clustering, six cell subgroups (M0-5) (Figure 2a) were identified from the Monocyte/Macrophage/DC population. M0 and M1 subgroups were found to highly express S100A8, S100A9, and S100A12, implying their role in antigen recognition and signal transduction, thus they were classified as classic $\mathrm{CD} 14^{+}$monocytes $(20,21)$ (Figure 2b, c). Other evidence in M1 came from the highly expressed genes of chemokines and cytokines such as CXCL8, CCL3, IL1B, and CXCL2(20, 21) (Figure 2c). M2 subgroup was found to express HES1, HES4, and ATF3 genes associated with regulation of transcription $(22,23)$ and were classified as non-classic CD16 ${ }^{+}$monocytes. M3 constituted fewer genes, mainly TAOK1, LINC01681, and SPATA22 with unspecified roles and requires further exploration. The significantly higher expression of FCGR3A(CD16) in M4 subgroup (Figure 2c) along with expression of IFN-related genes IFITM2 and IFITM3 suggests that M4 has genes with similar roles, as were found in subgroup M2 and were classified as $\mathrm{CD}^{+} 6^{+}$monocytes $(20,21)$. M5 subgroup consisted of genes involved in HLA-type recognition genes and were classified as $\mathrm{CD}^{+} \mathrm{C}^{+} \mathrm{DC}(21,24)$ (Figure 2c) .

\section{T/NK cell population is divided into two clusters based on their functions}

$\mathrm{T}$ cells are broadly classified into two subsets namely $\mathrm{CD} 4^{+} \mathrm{T}$ cells and $\mathrm{CD} 8^{+} \mathrm{T}$ cells based on their expression of markers and functions. In the present study, $\mathrm{CD} 4^{+}$and $\mathrm{CD} 8^{+}$cells were categorized as cluster 2 and cluster 1 respectively (Figure 1a) and were further subdivided into 9 subgroups (T0-8) (Figure 3a) . T0 subclusters contained genes expressing CD2, CD3D, CD3G and low CD8A along with significant expression of NKG7, GZMH and GZMB, a classic NK cell feature, and were therefore classified as cytotoxic NKT cells(Figure 3b, c, d) (25). T1 subcluster consisted of genes expressing IL7R, MAL, and CCR7, 
an indicator of $\mathrm{CD}^{+}{ }^{+} \mathrm{T}$ cell maturation (Figure $\mathbf{3 b}$, d) $(24,26,27)$. T2 subcluster consisted of GZMB, GZMA, high GNLY, NKG7, and FCGR3A (CD16) with low CD3D and CD3G expression and were classified as CD56 ${ }^{\mathrm{dim}} \mathrm{CD}^{+} 6^{+} \mathrm{NK}$ cells(Figure $\left.3 \mathbf{b}, \mathbf{c}\right)(25,28,29)$. T3 consisted of IL7R, MAL, CCR7 genes with significantly higher CD69 expression, indicating more active $\mathrm{CD} 4^{+} \mathrm{T}$ cell (Figure 3d) $(24,26,27,30)$. T4 consisted of genes featuring NK cell markers (NKG7 and GNLY) and genes with role in antigen recognition and presentation (LYZ, S100A8, and S100A9) and were identified as NK cell-derived antigen-presenting cells (APCs) (Figure 3b, d) (28, 29). T5 was considered as an unknown group with fewer genes. T6 were identified as NK cells (low GNLY, NKG7; high NKTR) with high XIST1 and SYNE2 indicating autophagic NK cells with low ribosomal expression (Figure 3b, d) (28, 29). Similar to the previous NK cells, T7 subgroup (GNLY, NKG7, high CD7, XCL1, and XCL2) was classified as cytokine induced memory like NK cells (CIML)(Figure 3d) (28, 29) and T8 (high GNLY and NKG7, IFIT2, IFIT3, IFNG, and PMAIP1) was identified as cytokine secreting CD $56^{\text {neg }}$ NK cells (Figure 3b) $(28,29)$.

Naive B cells constitute major B cell population in TA patients

Cluster 3 (B cell) and Cluster 9 (pDC) were further subdivided into 5 B cell subgroups (B0-4) for analysis (Figure 4a) . B0 (IGKC) were classified as naïve B cells (Figure 4b, c) (20) . B1 subcluster also contained genes with low expression except IGLC2, IGLC3, other immunoglobulin genes and were also categorized as Naive B cells (20). B2 subclusters were classified as plasmacytoid DCs (pDCs) (Figure 4c) (20, 21) with genes responsible for signal transduction and cytokine secretion (ITM2C, IRF7, LILRA4, and CCDC50). B3 was classified as Plasma B-cells (Figure 4c )(31) and contained genes (BPTF, NEAT1, and GOLGA4) regulating transcription, protein transport, and protein modification(32-34) and B4 was classified as unknown.

$\mathrm{CD}^{+} 4^{+}$monocyte, $\mathrm{GZMB}^{+} \mathrm{CD}^{+} \mathrm{T}$ cell, $\mathrm{CD}^{+{ }^{\mathrm{dim}} \mathrm{CD}^{+} 6^{+}} \mathrm{NK}$ cell, and naïve $\mathrm{B}$ cell were increased significantly in TA patients' PBMC

According to the cluster analysis in this study, we compared the proportions of different types of cells in the TA patients and healthy controls. We used $\chi^{2}$ test to analyze the differences in the composition of M, T, and B cell populations between TA patients and healthy controls by SPSS 20.0. The data showed that M (Cluster $0,4,6,8)$ and $\mathrm{B}$ (Cluster 3,9) cells increased significantly while $\mathrm{T}$ (Cluster 1,2) cell population decreased $(\mathrm{P}<0.05)$ (Table 2, Figure $\mathrm{S} 1)$ in TA patients as compared to healthy controls.

In order to further analyze the differences in gene expression of TA patients and healthy controls, we compared the composition of M, T, and B cell subclusters (Figure S2a, b, c) . Major findings of our study showed a significant increase in $\mathrm{CD} 14^{+}$monocytes and a reduction in $\mathrm{CD} 16^{+}$monocytes to $\mathrm{CD} 1 \mathrm{C}^{+} \mathrm{DCs}$ ratio in $\mathrm{M}$ cell types $(\mathrm{P}<0.05)$. Among the $\mathrm{T}$ cell populations, the proportion of NKT cells and CD56 ${ }^{\mathrm{dim}} \mathrm{CD} 16^{+}$ NK cells increased while proportion of $\mathrm{CD}^{+} \mathrm{T}$ cells, APCs, low ribosomal expressing NK cells, CIML, and CD56 $6^{\text {neg }} \mathrm{NK}$ cells decreased in TA patients as compared to healthy controls $(\mathrm{P}<0.05)$. In the $\mathrm{B}$ cell population, the ratio of naïve $\mathrm{B}$ cells increased while the ratio of plasma cells to pDCs decreased in TA patients as compared to healthy controls $(\mathrm{P}<0.05)$.

M, T, B cells have different differentially expressed genes (DEGs) between TA patients and healthy controls

Peripheral blood of TA patients and healthy controls showed different DEGs for different cell types (Figure S3). The expression of different markers were compared and were found to be significantly higher in TA patients as compared to healthy controls in M cells (IL1R2, THBS1, AREG, CD163, and FKBP5) (Figure S3a) ; T cells (IFITM1, FKBP5, MIF, and TXNIP) (Figure S3b) and B cell groups (TXNIP, IGHG1, MIF, and IGHG3) (Figure S3c) .

\section{Discussion}

scRNA-seq can more accurately analyze each cell's type and functions by sequencing their own transcriptome compared to bulk RNA-seq. In this study, scRNA-seq was used to detect the inflammatory cells types and cell markers in the peripheral blood of TA patients. 
$\mathrm{CD}^{+} \mathrm{T}$ cells have always been the focus of TA research, and its differentiation subtypes, Th1 and Th17 cells are considered to be the main regulators of inflammation in TA(13). Our study found fewer CD4 ${ }^{+}$ $\mathrm{T}$ cells in the peripheral blood of TA patients as compared to the healthy controls and can be an effect of glucocorticoids and other immunosuppressive drugs taken by the patients for TA $(35,36)$. We also saw an increase in cytotoxic NKT cells and NK cells in TA patients indicating that these cells have the ability to regulate inflammation and can play a major role in vascular damage associated with TA. Our study further suggests that limiting the number of these cells may effectively control the disease progression in TA patients. In recent years, monocyte/macrophages have been extensively studied for their role in immune diseases. In the present study, we see a higher proportion of monocyte/macrophages represented by CD14 ${ }^{+}$ in TA patients indicating that these cells may play an important role in TA. B cells have always been less involved in TA research, but in recent years, more studies indicating the role of B cell in TA pathogenesis have been published. Many studies(37-42) have showed B cells have played an important role in the TA lesions during the courses of the disease, however, the specific role of B cells in TA is still unclear. In our study, we found that the proportion of B cells (especially naive B cells) were higher in TA patients, indicating that both cellular and humoral immunity plays a role in TA pathogenesis.

We further analyzed the cell markers of different cell types that are abnormally expressed in TA. In the monocyte/macrophage subset of TA patients, we found an increase in the expression of IL1R2, THBS1, CD163, AREG, and FKBP5. Among these 5 genes, IL1R2(43) and FKBP5(44) can be elevated due to the intake of glucocorticoids. CD163 is a transmembrane scavenger receptor that is expressed on the surface of macrophages(45) and is known to be elevated in SLE, sJIA, and Kawasaki patients, indicating that CD163 can be used as a marker for macrophage activation syndrome (MAS) indicating the transition from monocyte/macrophage to M1 proinflammatory macrophages $(46,47)$. In the present study, the elevated CD163 gene in TA patients indicates that these cells are in an active state of inflammation, suggesting that monocytes/macrophages play an important role in the pathogenesis of TA, suggesting that CD163 may be used as a potential diagnostic marker for TA as well. THBS1, also known as thrombospondin 1 (TSP1), is a ligand for CD47. This protein exists in the extracellular matrix and can promote tissue fibrosis by binding fibrin and collagen(48). In rheumatoid arthritis patients, THBS1 activates the inflammatory function of $\mathrm{T}$ cells through the CD47 receptor on the surface of T cells $(49,50)$, and activates macrophages via the Toll-like receptor 4 (TLR4) pathway $(51,52)$. This protein can also inhibit the activity of VEGF in tumor-related research, thereby inhibiting the synthesis of new blood vessels $(52,53)$. AREG is one of the main ligands of the EFGR pathway. The protein is mainly used to regulate the proliferation, apoptosis, and metastasis of various cells(54). Under pathological conditions, especially in patients with chronic diseases, such as cirrhosis(55), chronic obstructive pulmonary disease (COPD)(56), the expression of AREG is significantly elevated. In recent studies on mouse models of glomerulonephritis, AREG has been shown to play a completely different role in $\mathrm{CD} 4^{+} \mathrm{T}$ cells and macrophages. This protein enhances the function of Treg cells and inhibits the growth of $\mathrm{CD}^{+} \mathrm{T}$ cells and promotes the recruitment of myeloid cells and the proliferation and cytokine secretion of M1 cells(57).

In the T cells of TA patients, we found a significant increase in the expression of IFITM1, FKBP5, MIF, and TXNIP. Similar to IL1R2 and FKBP5, the increase in MIF is closely related to the intake of glucocorticoids(58). The patients in this study were all taking glucocorticoids, which is not discussed here. IFITM1 is an IFN-related protein with a role in inhibiting viral infection by interfering with viral protein synthesis and replication(59, 60). IFITM1 is mainly expressed on T cell surface. In addition to the above functions, IFITM1 can also promote the differentiation of naive $\mathrm{CD} 4^{+} \mathrm{T}$ cells into Th2 cells(61), indicating that this protein is involved in inflammatory regulation. TXNIP is a binding protein of thioredoxin (TXN), and can inhibit the antioxidant capacity of TXN and promote cell stress(62). In the inflammatory process, TXNIP can promote the formation of ROS-NLRP3 inflammasomes by inhibiting the transfer of reactive oxygen species (ROS) by TXN, thereby increasing the concentration of IL-18 and IL-1 $\beta(63)$. This inflammatory reaction process also occurs in patients with coronary artery disease(64) and diabetes-related vascular disease(65). An increase in IL-18 in peripheral blood of TA patients has been shown to promote the formation of granulomas(66).

Our findings showed a similar increase in the expression of IGHG1, IGHG3, MIF, and TXNIP in B cells of 
TA patients suggesting a role of TXNIP in TA pathogenesis. The immunoglobulin heavy constant G chain (IGHG) genes are known to play a crucial role in the synthesis of immunoglobulins by B cells(67). Related reports of IGHG have also been found in other autoimmune diseases $(68,69)$. The increased expression of this gene in PBMCs of TA patients in our study indicates the secretion of antibodies and the role of humoral immunity in the pathogenesis of TA.

According to the previous studies mentioned before, it can be found that THBS1, CD163, AREG, IFITM1, TXNIP, and IGHGs expression are all related to the differentiation and activity of inflammatory cells. Therefore, the expression level of these cell markers can reflect the state of inflammatory cells, in addition the disease activity of TA patients.

In conclusion, we used single-cell RNAseq technology to detect peripheral blood cells in TA patients. Our study showed that CD14 ${ }^{+}$monocytes, cytotoxic NKT cells, CD $56^{\mathrm{dim}} \mathrm{CD} 16^{+} \mathrm{NK}$ cells, and B cells were elevated in the peripheral blood of TA patients suggesting CD4 ${ }^{+} \mathrm{T}$ cell and IL6 pathway are not the only key, these kinds of cells are also playing a crucial role in TA pathogenesis and the potential use of THBS1, CD163, AREG, IFITM1, TXNIP, and IGHGs expression as diagnostic markers for TA development and progression.

\section{Author contributions}

G.Q., L.W., L.Y. designed the study; G.Q. did the experiments. Y.Jinge, Y.Jinfeng and G.Q. analyzed the scRNA-seq data. C.Z., D.Y., M.Y. and J.J provided human blood sample and checked the case history. L.W., L.Y. wrote the paper.

\section{Funding}

This work was supported by a grant from National Science and Technology Major Project of China (2018ZX10302 302001004); National Natural Science Foundation of China (U1903118); Beijing Hospital Clinical Research 121 project (no. BJ-2018-089) and the Fundamental Research Funds for the Central Universities (no. 3332018174).

\section{Ethics Statement}

This work was reviewed and approved by Beijing Hospital Ethics Committee (no. 2018BJYYEC-030-01).

\section{Data availability}

All the scRNA-seq expression matrix and cases clinical data are available from authors.

\section{Conflict of interest}

All authors declare no conflict of interest.

\section{References}

1. Kerr GS, Hallahan CW, Giordano J, Leavitt RY, Fauci AS, Rottem M, et al. Takayasu arteritis. Ann Intern Med. 1994;120(11):919-29.

2. Comarmond C, Biard L, Lambert M, Mekinian A, Ferfar Y, Kahn JE, et al. Long-Term Outcomes and Prognostic Factors of Complications in Takayasu Arteritis: A Multicenter Study of 318 Patients. Circulation. 2017;136(12):1114-22.

3. Hoffman GS, Leavitt RY, Kerr GS, Rottem M, Sneller MC, Fauci AS. Treatment of glucocorticoid-resistant or relapsing Takayasu arteritis with methotrexate. Arthritis Rheum. 1994;37(4):578-82.

4. Li J, Yang Y, Zhao J, Li M, Tian X, Zeng X. The efficacy of Mycophenolate mofetil for the treatment of Chinese Takayasu's arteritis. Sci Rep. 2016;6:38687.

5. Dai D, Wang Y, Jin H, Mao Y, Sun H. The efficacy of mycophenolate mofetil in treating Takayasu arteritis: a systematic review and meta-analysis. Rheumatol Int. 2017;37(7):1083-8. 
6. Nakaoka Y, Isobe M, Takei S, Tanaka Y, Ishii T, Yokota S, et al. Efficacy and safety of tocilizumab in patients with refractory Takayasu arteritis: results from a randomised, double-blind, placebo-controlled, phase 3 trial in Japan (the TAKT study). Ann Rheum Dis. 2018;77(3):348-54.

7. Langford CA, Cuthbertson D, Ytterberg SR, Khalidi N, Monach PA, Carette S, et al. A Randomized, Double-Blind Trial of Abatacept (CTLA-4Ig) for the Treatment of Takayasu Arteritis. Arthritis Rheumatol. 2017;69(4):846-53.

8. Chen ZG, Chen YX, Diao YP, Wu ZY, Yan S, Ma L, et al. Simultaneous Multi-Supra-Aortic Artery Bypass Successfully Implemented in 17 Patients with Type I Takayasu Arteritis. Eur J Vasc Endovasc Surg. 2018;56(6):903-9.

9. Diao Y, Yan S, Premaratne S, Chen Y, Tian X, Chen Z, et al. Surgery and Endovascular Management in Patients With Takayasu's Arteritis: A Ten-Year Retrospective Study. Ann Vasc Surg. 2020;63:34-44.

10. Kinjo H, Kafa A. The results of treatment in renal artery stenosis due to Takayasu disease: comparison between surgery, angioplasty, and stenting. A monocentrique retrospective study. G Chir. 2015;36(4):161-7.

11. Gulcu A, Gezer NS, Akar S, Akkoc N, Onen F, Goktay AY. Long-Term Follow-Up of Endovascular Repair in the Management of Arterial Stenosis Caused by Takayasu's Arteritis. Ann Vasc Surg. 2017;42:93-100.

12. Park HS, Do YS, Park KB, Kim DK, Choo SW, Shin SW, et al. Long term results of endovascular treatment in renal arterial stenosis from Takayasu arteritis: angioplasty versus stent placement. Eur J Radiol. 2013;82(11):1913-8.

13. Saadoun D, Garrido M, Comarmond C, Desbois AC, Domont F, Savey L, et al. Th1 and Th17 cytokines drive inflammation in Takayasu arteritis. Arthritis Rheumatol. 2015;67(5):1353-60.

14. Sagar S, Ganguly NK, Koicha M, Sharma BK. Immunopathogenesis of Takayasu arteritis. Heart Vessels Suppl. 1992;7:85-90.

15. Misra DP, Chaurasia S, Misra R. Increased Circulating Th17 Cells, Serum IL-17A, and IL-23 in Takayasu Arteritis. Autoimmune Dis. 2016;2016:7841718.

16. Papalexi E, Satija R. Single-cell RNA sequencing to explore immune cell heterogeneity. Nat Rev Immunol. 2018;18(1):35-45.

17. See P, Lum J, Chen J, Ginhoux F. A Single-Cell Sequencing Guide for Immunologists. Front Immunol. 2018;9:2425.

18. Potter SS. Single-cell RNA sequencing for the study of development, physiology and disease. Nat Rev Nephrol. 2018;14(8):479-92.

19. Fernandez DM, Rahman AH, Fernandez NF, Chudnovskiy A, Amir ED, Amadori L, et al. Single-cell immune landscape of human atherosclerotic plaques. Nat Med. 2019;25(10):1576-88.

20. Zhao J, Zhang S, Liu Y, He X, Qu M, Xu G, et al. Single-cell RNA sequencing reveals the heterogeneity of liver-resident immune cells in human. Cell Discov. 2020;6:22.

21. Villani AC, Satija R, Reynolds G, Sarkizova S, Shekhar K, Fletcher J, et al. Single-cell RNA-seq reveals new types of human blood dendritic cells, monocytes, and progenitors. Science. 2017;356(6335).

22. Rani A, Greenlaw R, Smith RA, Galustian C. HES1 in immunity and cancer. Cytokine Growth Factor Rev. 2016;30:113-7.

23. Ku HC, Cheng CF. Master Regulator Activating Transcription Factor 3 (ATF3) in Metabolic Homeostasis and Cancer. Front Endocrinol (Lausanne). 2020;11:556. 
24. Kazer SW, Aicher TP, Muema DM, Carroll SL, Ordovas-Montanes J, Miao VN, et al. Integrated singlecell analysis of multicellular immune dynamics during hyperacute HIV-1 infection. Nat Med. 2020;26(4):5118.

25. Zhou L, Adrianto I, Wang J, Wu X, Datta I, Mi QS. Single-Cell RNA-Seq Analysis Uncovers Distinct Functional Human NKT Cell Sub-Populations in Peripheral Blood. Front Cell Dev Biol. 2020;8:384.

26. Schafflick D, Xu CA, Hartlehnert M, Cole M, Schulte-Mecklenbeck A, Lautwein T, et al. Integrated single cell analysis of blood and cerebrospinal fluid leukocytes in multiple sclerosis. Nat Commun. 2020;11(1):247.

27. Zemmour D, Zilionis R, Kiner E, Klein AM, Mathis D, Benoist C. Single-cell gene expression reveals a landscape of regulatory T cell phenotypes shaped by the TCR. Nat Immunol. 2018;19(3):291-301.

28. Yang C, Siebert JR, Burns R, Gerbec ZJ, Bonacci B, Rymaszewski A, et al. Heterogeneity of human bone marrow and blood natural killer cells defined by single-cell transcriptome. Nat Commun. 2019;10(1):3931.

29. Smith SL, Kennedy PR, Stacey KB, Worboys JD, Yarwood A, Seo S, et al. Diversity of peripheral blood human NK cells identified by single-cell RNA sequencing. Blood Adv. 2020;4(7):1388-406.

30. Cibrian D, Sanchez-Madrid F. CD69: from activation marker to metabolic gatekeeper. Eur J Immunol. 2017;47(6):946-53.

31. Hanamsagar R, Reizis T, Chamberlain M, Marcus R, Nestle FO, de Rinaldis E, et al. An optimized workflow for single-cell transcriptomics and repertoire profiling of purified lymphocytes from clinical samples. Sci Rep. 2020;10(1):2219.

32. Landry J, Sharov AA, Piao Y, Sharova LV, Xiao H, Southon E, et al. Essential role of chromatin remodeling protein Bptf in early mouse embryos and embryonic stem cells. PLoS Genet. 2008;4(10):e1000241.

33. Prinz F, Kapeller A, Pichler M, Klec C. The Implications of the Long Non-Coding RNA NEAT1 in Non-Cancerous Diseases. Int J Mol Sci. 2019;20(3).

34. Wong M, Munro S. Membrane trafficking. The specificity of vesicle traffic to the Golgi is encoded in the golgin coiled-coil proteins. Science. 2014;346(6209):1256898.

35. Ashwell JD, Lu FW, Vacchio MS. Glucocorticoids in T cell development and function*. Annu Rev Immunol. 2000;18:309-45.

36. Taves MD, Ashwell JD. Glucocorticoids in T cell development, differentiation and function. Nat Rev Immunol. 2020.

37. Clement M, Galy A, Bruneval P, Morvan M, Hyafil F, Benali K, et al. Tertiary Lymphoid Organs in Takayasu Arteritis. Front Immunol. 2016;7:158.

38. Kurata A, Saito A, Hashimoto H, Fujita K, Ohno SI, Kamma H, et al. Difference in immunohistochemical characteristics between Takayasu arteritis and giant cell arteritis: It may be better to distinguish them in the same age. Mod Rheumatol. 2019;29(6):992-1001.

39. Hoyer BF, Mumtaz IM, Loddenkemper K, Bruns A, Sengler C, Hermann KG, et al. Takayasu arteritis is characterised by disturbances of B cell homeostasis and responds to B cell depletion therapy with rituximab. Ann Rheum Dis. 2012;71(1):75-9.

40. Wang H, Ma J, Wu Q, Luo X, Chen Z, Kou L. Circulating B lymphocytes producing autoantibodies to endothelial cells play a role in the pathogenesis of Takayasu arteritis. J Vasc Surg. 2011;53(1):174-80.

41. Nishino Y, Tamai M, Kawakami A, Koga T, Makiyama J, Maeda Y, et al. Serum levels of BAFF for assessing the disease activity of Takayasu arteritis. Clin Exp Rheumatol. 2010;28(1 Suppl 57):14-7.

42. Zanwar A, Jain A, Gupta L, Chaurasia S, Kumar S, Misra DP, et al. Serum BAFF and APRIL levels in Indian patients with Takayasu arteritis. Clin Rheumatol. 2018;37(12):3439-42. 
43. Colotta F, Saccani S, Giri JG, Dower SK, Sims JE, Introna M, et al. Regulated expression and release of the IL-1 decoy receptor in human mononuclear phagocytes. J Immunol. 1996;156(7):2534-41.

44. Binder EB. The role of FKBP5, a co-chaperone of the glucocorticoid receptor in the pathogenesis and therapy of affective and anxiety disorders. Psychoneuroendocrinology. 2009;34 Suppl 1:S186-95.

45. Law SK, Micklem KJ, Shaw JM, Zhang XP, Dong Y, Willis AC, et al. A new macrophage differentiation antigen which is a member of the scavenger receptor superfamily. Eur J Immunol. 1993;23(9):2320-5.

46. Porcheray F, Viaud S, Rimaniol AC, Leone C, Samah B, Dereuddre-Bosquet N, et al. Macrophage activation switching: an asset for the resolution of inflammation. Clin Exp Immunol. 2005;142(3):481-9.

47. Avcin T, Tse SM, Schneider R, Ngan B, Silverman ED. Macrophage activation syndrome as the presenting manifestation of rheumatic diseases in childhood. J Pediatr. 2006;148(5):683-6.

48. Isenberg JS, Roberts DD. THBS1 (thrombospondin-1). Atlas Genet Cytogenet Oncol Haematol. 2020;24(8):291-9.

49. Vallejo AN, Yang H, Klimiuk PA, Weyand CM, Goronzy JJ. Synoviocyte-mediated expansion of inflammatory $\mathrm{T}$ cells in rheumatoid synovitis is dependent on CD47-thrombospondin 1 interaction. J Immunol. 2003;171(4):1732-40.

50. Hu F, Liu H, Xu L, Li Y, Liu X, Shi L, et al. Hypoxia-inducible factor-1alpha perpetuates synovial fibroblast interactions with T cells and B cells in rheumatoid arthritis. Eur J Immunol. 2016;46(3):742-51.

51. Li Y, Qi X, Tong X, Wang S. Thrombospondin 1 activates the macrophage Toll-like receptor 4 pathway. Cell Mol Immunol. 2013;10(6):506-12.

52. Murphy-Ullrich JE. Thrombospondin 1 and Its Diverse Roles as a Regulator of Extracellular Matrix in Fibrotic Disease. J Histochem Cytochem. 2019;67(9):683-99.

53. Lawler PR, Lawler J. Molecular basis for the regulation of angiogenesis by thrombospondin-1 and -2 . Cold Spring Harb Perspect Med. 2012;2(5):a006627.

54. Berasain C, Avila MA. Amphiregulin. Semin Cell Dev Biol. 2014;28:31-41.

55. Perugorria MJ, Latasa MU, Nicou A, Cartagena-Lirola H, Castillo J, Goni S, et al. The epidermal growth factor receptor ligand amphiregulin participates in the development of mouse liver fibrosis. Hepatology. 2008;48(4):1251-61.

56. Val S, Belade E, George I, Boczkowski J, Baeza-Squiban A. Fine PM induce airway MUC5AC expression through the autocrine effect of amphiregulin. Arch Toxicol. 2012;86(12):1851-9.

57. Melderis S, Hagenstein J, Warkotsch MT, Dang J, Herrnstadt GR, Niehus CB, et al. Amphiregulin Aggravates Glomerulonephritis via Recruitment and Activation of Myeloid Cells. J Am Soc Nephrol. 2020;31(9):1996-2012.

58. Kang I, Bucala R. The immunobiology of MIF: function, genetics and prospects for precision medicine. Nat Rev Rheumatol. 2019;15(7):427-37.

59. Bailey CC, Zhong G, Huang IC, Farzan M. IFITM-Family Proteins: The Cell's First Line of Antiviral Defense. Annu Rev Virol. 2014;1:261-83.

60. Raychoudhuri A, Shrivastava S, Steele R, Kim H, Ray R, Ray RB. ISG56 and IFITM1 proteins inhibit hepatitis C virus replication. J Virol. 2011;85(24):12881-9.

61. Yu Q, Sharma A, Oh SY, Moon HG, Hossain MZ, Salay TM, et al. T cell factor 1 initiates the T helper type 2 fate by inducing the transcription factor GATA-3 and repressing interferon-gamma. Nat Immunol. 2009;10(9):992-9. 
62. Tinkov AA, Bjorklund G, Skalny AV, Holmgren A, Skalnaya MG, Chirumbolo S, et al. The role of the thioredoxin/thioredoxin reductase system in the metabolic syndrome: towards a possible prognostic marker? Cell Mol Life Sci. 2018;75(9):1567-86.

63. Kim SK, Choe JY, Park KY. TXNIP-mediated nuclear factor-kappaB signaling pathway and intracellular shifting of TXNIP in uric acid-induced NLRP3 inflammasome. Biochem Biophys Res Commun. 2019;511(4):725-31.

64. Rong J, Xu X, Xiang Y, Yang G, Ming X, He S, et al. Thioredoxin-interacting protein promotes activation and inflammation of monocytes with DNA demethylation in coronary artery disease. J Cell Mol Med. 2020;24(6):3560-71.

65. Amin FM, Abdelaziz RR, Hamed MF, Nader MA, Shehatou GSG. Dimethyl fumarate ameliorates diabetes-associated vascular complications through ROS-TXNIP-NLRP3 inflammasome pathway. Life Sci. 2020;256:117887.

66. Alibaz-Oner F, Yentur SP, Saruhan-Direskeneli G, Direskeneli H. Serum cytokine profiles in Takayasu's arteritis: search for biomarkers. Clin Exp Rheumatol. 2015;33(2 Suppl 89):S-32-5.

67. Oxelius VA, Pandey JP. Human immunoglobulin constant heavy G chain (IGHG) (Fcgamma) (GM) genes, defining innate variants of IgG molecules and B cells, have impact on disease and therapy. Clin Immunol. 2013;149(3):475-86.

68. Oxelius VA, Svantesson H, Carlsson AM. Gm phenotype linkage to subsets of juvenile chronic arthritis (JCA) with influence on IgG subclass response. Scand J Rheumatol. 1993;22(6):284-8.

69. Jonsson G, Oxelius VA, Truedsson L, Braconier JH, Sturfelt G, Sjoholm AG. Homozygosity for the IgG2 subclass allotype G2M(n) protects against severe infection in hereditary C2 deficiency. J Immunol. 2006;177(1):722-8.

70. Stuart T, Butler A, Hoffman P, Hafemeister C, Papalexi E, Mauck WM, 3rd, et al. Comprehensive Integration of Single-Cell Data. Cell. 2019;177(7):1888-902 e21.

71. Butler A, Hoffman P, Smibert P, Papalexi E, Satija R. Integrating single-cell transcriptomic data across different conditions, technologies, and species. Nat Biotechnol. 2018;36(5):411-20.

Table 1 cell marker of each cluster

\begin{tabular}{lll}
\hline Cluster & Cell type & Cell marker \\
\hline $0,4,6,8$ & $\mathrm{M}$ & S100A8, LYZ, S100A9 \\
1,2 & $\mathrm{~T}$ & CD2, CD3D, NKG7, GNLY \\
3,9 & $\mathrm{~B}$ & MS4A1, CD79A, CD79B, ITM2C, TCF4, CCDC50 \\
7 & megakaryocyte & PF4, PPBP \\
5 & & NEAT1, XIST \\
\hline
\end{tabular}

Table 2 Cell count of each type

\begin{tabular}{llllll}
\hline & mono & T cell & B cell & mega & unknown \\
\hline TA & 5042 & 2196 & 1390 & 44 & 294 \\
HC & 10144 & 6771 & 2709 & 290 & 1062 \\
P value & $¡ 0.05$ & $¡ 0.05$ & $¡ 0.05$ & $¡ 0.05$ & $¡ 0.05$ \\
\hline
\end{tabular}

Table S1 Baseline of scRNA-seq cases 


\begin{tabular}{llllllll}
\hline Number & Gender & Age & ESR & CRP & Prednisone $(\mathrm{mg} / \mathrm{d})$ & Methylprednisolone $(\mathrm{mg} / \mathrm{d})$ & Methotrexate $(\mathrm{mg} / \mathrm{d})$ \\
\hline TA1 & Female & 39 & 5 & $i 0.1$ & 0 & 16 & 15 \\
TA2 & Female & 32 & 5 & 0.1 & 0 & 20 & 12.5 \\
TA3 & Female & 16 & 7 & 1.1 & 17.5 & 0 & 10 \\
TA4 & Female & 24 & 7 & 0.2 & 0 & 8 & 0 \\
\hline
\end{tabular}

Table S2. Cell count of each sample

\begin{tabular}{llllllllll}
\hline & TA & TA & TA & TA & Control & Control & Control & Control & Total \\
& B1 & B2 & B3 & B4 & H1 & H2 & H3 & H4 & \\
Count & 3802 & 2913 & 1373 & 878 & 6369 & 4025 & 5752 & 4830 & 29942 \\
Total & 8966 & 8966 & 8966 & 8966 & 20976 & 20976 & 20976 & 20976 & \\
\hline
\end{tabular}



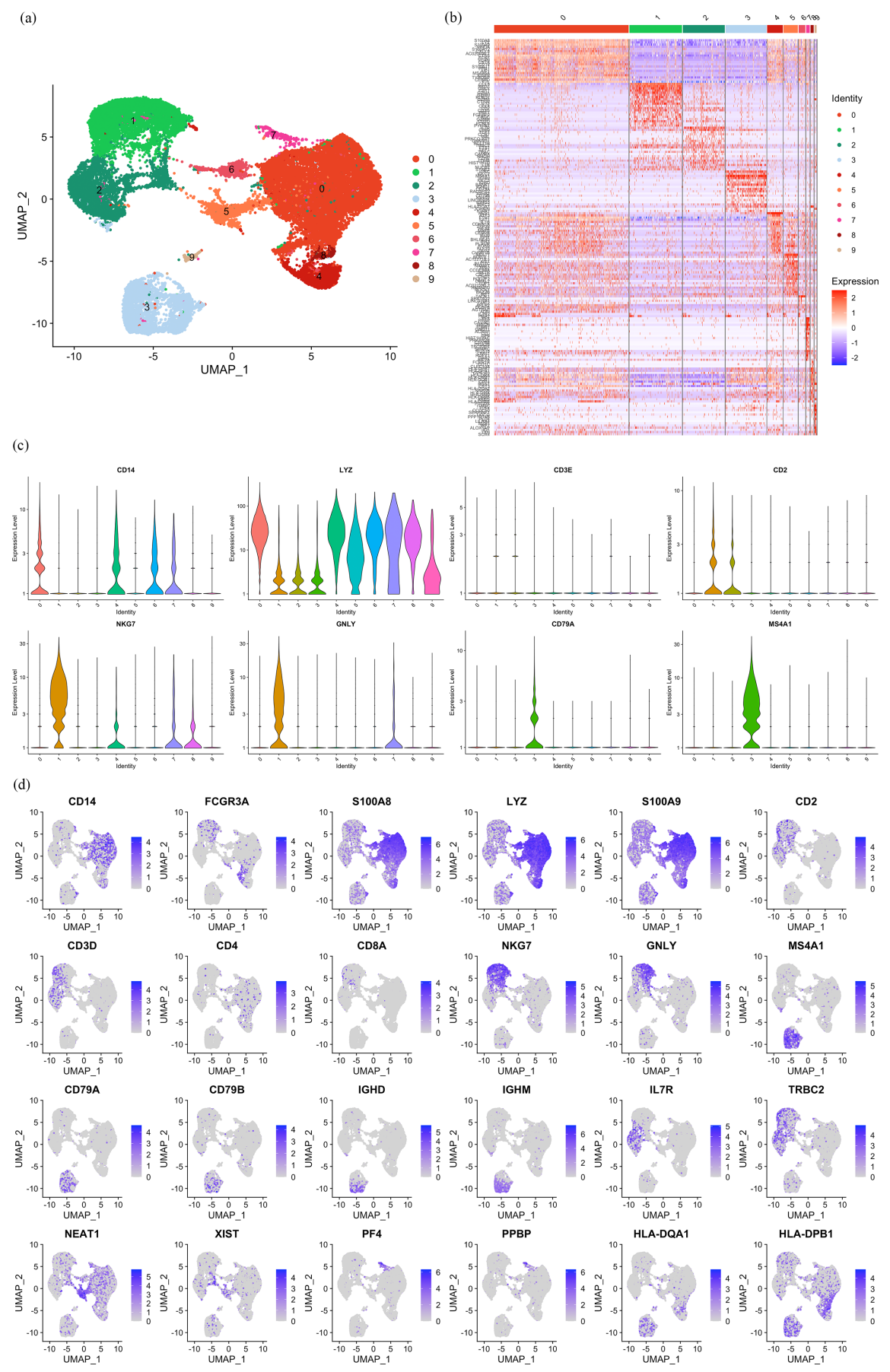

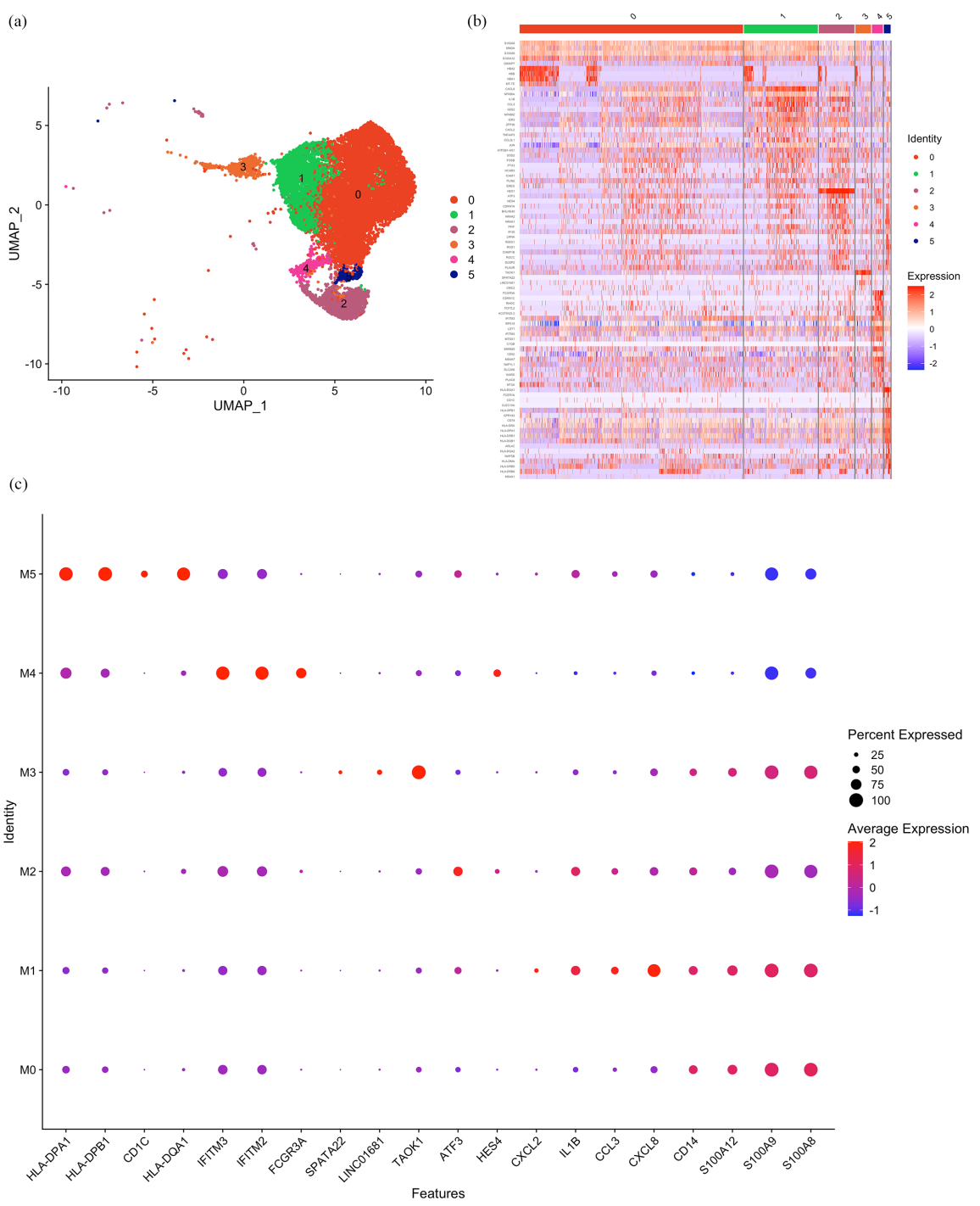

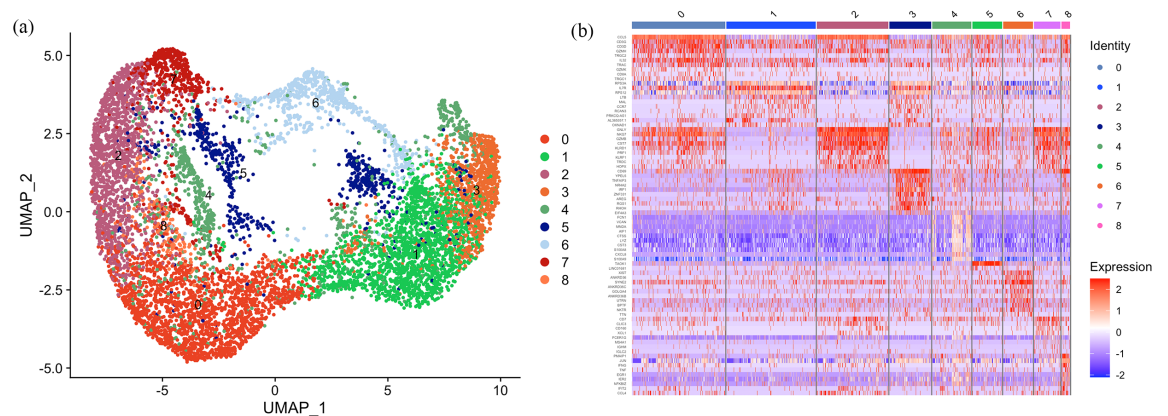

(c)
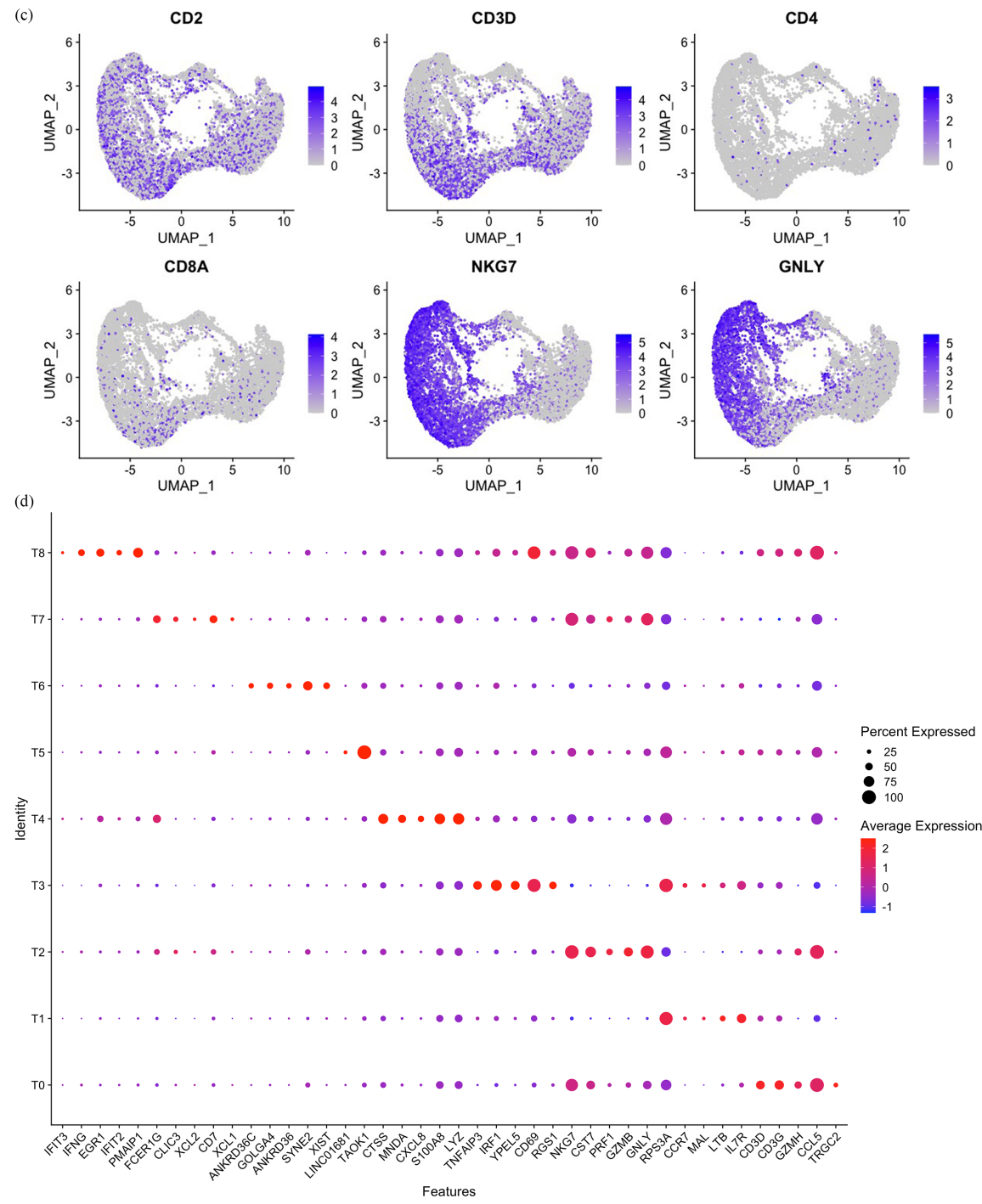


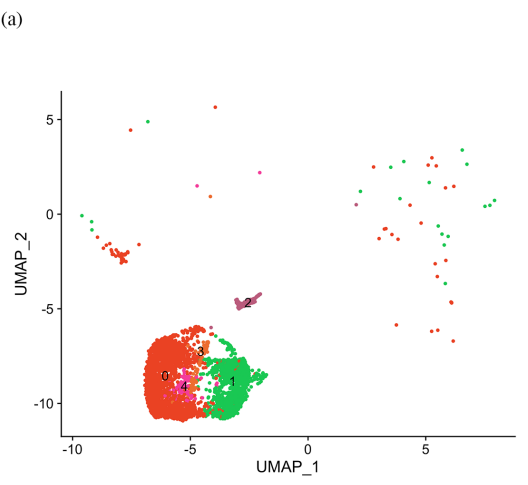

(c)
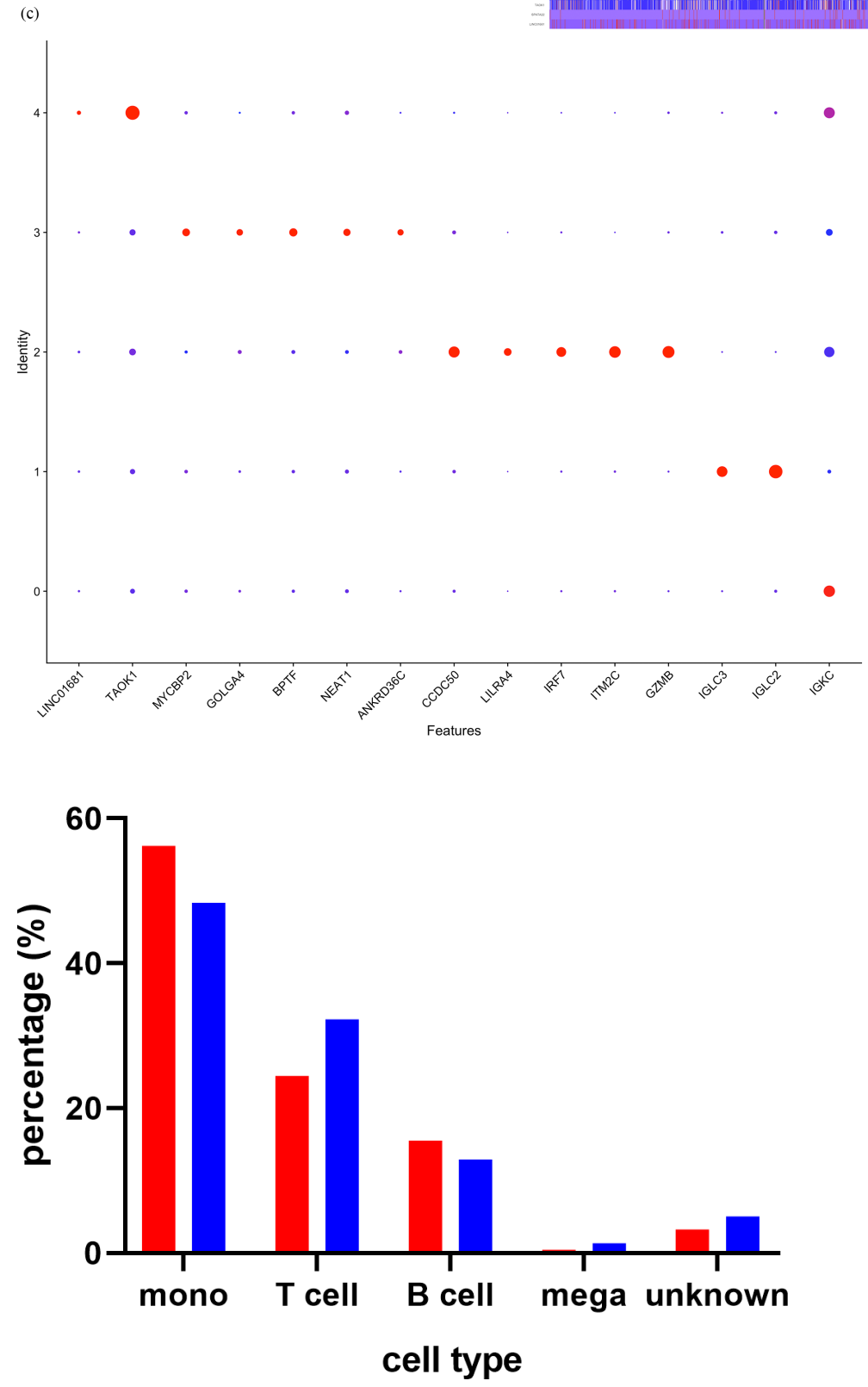

(b)
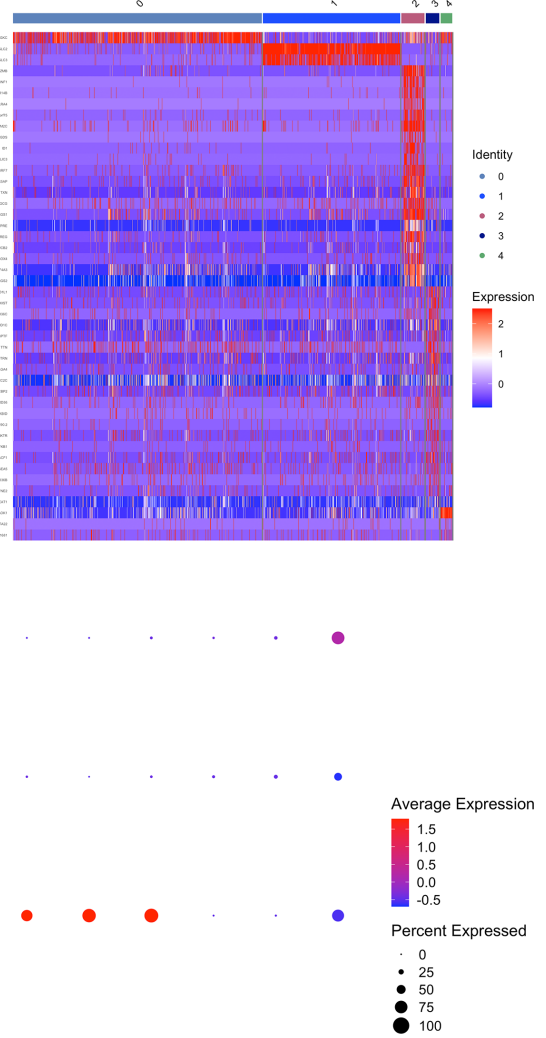

$$
\text { - }
$$

TA
$\mathrm{HC}$ 

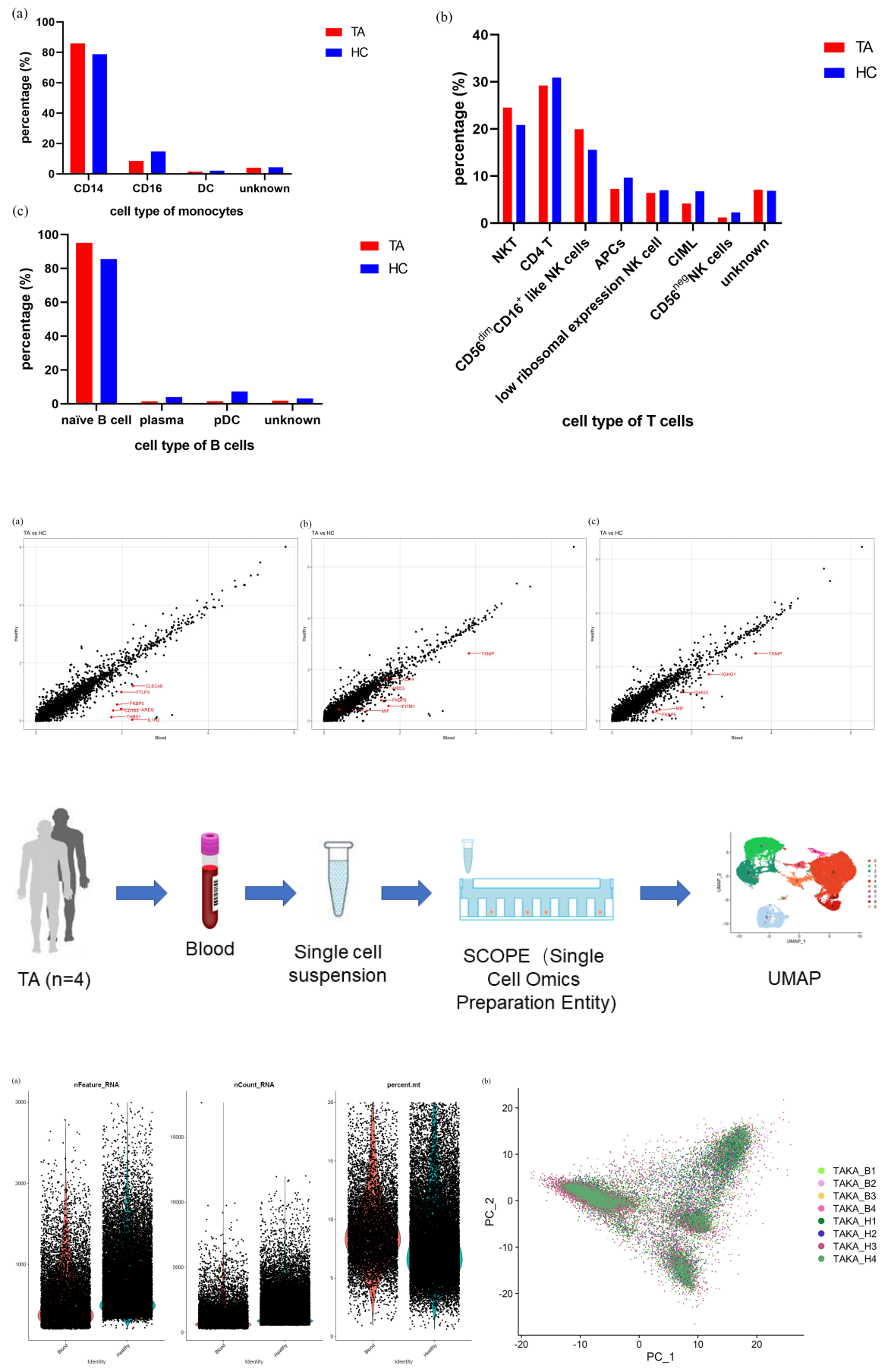